Do we need critical health psychology or rather critical health psychologists? Peer-reviewed author version

VINCK, Jan \& MEGANCK, Jeroen (2006) Do we need critical health psychology or rather critical health psychologists?. In: Journal of Health Psychology, 11(3). p. 391-393.

DOI: $10.1177 / 1359105306063310$

Handle: http://hdl.handle.net/1942/1354 


\title{
Do we need critical health psychology or rather critical health psychologists?
}

Jan Vinck \& Jeroen Meganck

Hasselt University, Health Psychology Research Group

\begin{abstract}
In her paper Julie Hepworth discusses the definition of critical health psychology and its position within the field of health psychology. We understand that she is inclined to favour a position of critical health psychology that is separate from and in opposition to mainstream health psychology. In contrast to this, we are convinced that the important concerns of critical health psychology are better served by efforts to help mainstream health psychologists to think and work more strongly from a critical perspective.
\end{abstract}

Keywords: Critical health psychology; health psychology; public health; professionalisation; interdisciplinary relations

In this commentary on the paper by Julie Hepworth on the emergence of critical health psychology and its potential contribution to public health (Hepworth 2006, in press), we want to discuss our impression that the author feels that critical health psychology (CHP) should develop as a separate discipline, apart from and to a certain extent in opposition to mainstream health psychology (MHP). We do so from our background as health psychologists working in a health promotion setting. From this applied perspective we look at the more theoretical discussions about what health psychology is, and how its different sub-disciplines should be differentiated.

Of course, defending that CHP should be a separate sub-discipline, is only one element from the author's statement in favour of a significant role for CHP. And of course she is correct when she underlines the many and important contributions that are presented as CHP's "major developments for health psychology”. These can be found at different levels: a strong focus on ethical considerations and an explicit “call for action”, promotion of the long neglected contextuality of behaviour including the cultural, political and economical contexts, the 
critique on a dualistic view of humanity, the revalorisation of qualitative research, etc. It would be foolish to underestimate the importance of these elements.

Having said this, the question remains as to how, in terms of the development of our profession, these contributions can best be given a place, so that the chance that they have a real impact, e.g. in the promotion of health and well-being, and in the advancement of scientific knowledge, is maximised. As was said in the beginning of this comment, we have the impression that the author favours the idea that this will best be guaranteed by the establishment of CHP as a separate discipline, continually operating as a kind of consciousness for, and a permanent and independent “critique” on MHP.

Before we discuss our concerns about this specific issue, we would like to draw attention to another aspect of the paper's position about the relation between CHP and MHP. We get the feeling from Julie's paper that CHP (or she?) tends to see the characteristics of CHP, described above, as positive evolutions (which we agree they are) in opposition to the "wrong” characteristics of MHP, and as characteristics that are exclusively found in CHP. Of course such contrasts are useful to make your point clearly, but they should definitely not be taken as absolute. It is true, e.g., that MHP has long neglected qualitative research in favour of quantitative methods, but it is equally evident that MHP has been learning for some time now how both approaches complement each other. Also, MHP has indeed had a tendency to isolate behaviour from its context, but authors reflecting the vision of the APA Division of Health Psychology - and who for this reason, we assume, are to be considered as representative for MHP - have recently argued that one of the developments that is necessary for the future of health psychology is a stronger emphasis on the training of "contextually competent" researchers and practitioners, and that research and practice have to increasingly include organizational, cultural, economical and political systems, thereby also answering the critique that health psychology was, before, indeed too exclusively restricted to an individualistic approach (Smith and Suls, 2004). Additional examples to show that the "CHP versus MHP relationship" should not be pictured too much in "black and white" can easily be envisioned. We think that the examples given suffice to demonstrate that MHP may well be less “conservative” and resistant to the incorporation of new insights than some might think. 
The second aspect of our concern has, as we said, to do with the feeling that, in this paper, Julie defends the position that CHP should develop as a separate discipline, apart from and even in opposition to MHP. After having given this impression most strongly in the first part of the paper, Julie herself discusses this near the end as one of the dilemmas in the development of CHP: the dilemma between the risk of isolating itself and the risk of becoming incorporated in MHP. We think that this is only a dilemma when the idea is that CHP should evolve into a specific and separate discipline. Besides the problems that Julie herself mentions, we fear that such a development will almost inevitably create "territorial wars" between sub-disciplines, against which the author herself warns in the discussion on the definition of CHP. We are convinced that there is a much more interesting way to serve the concerns that CHP correctly advances: rather than working towards the establishment of CHP as a separate discipline, we should promote a critical attitude in all health psychologists, including the so called mainstream health psychologists. Rather than a discipline, we need a critical attitude in health psychology. This critical attitudes includes at least the following aspects: (1) ethical (justice, equity, responsibility, scientific independence...), (2) ideological (power, poverty, theory vs. action...), (3) methodological (quantitative vs. qualitative, interpretivism...) (4) theoretical (biopsychosocial model, dualism, ecological perspective...) and (5) professional (collaboration with other health psychologists, scientists and systems, training...). To be sure, this critical attitude may not easily develop spontaneously. It may require a lot of effort, both in terms of getting these ideas in health psychology curricula, and of getting professional bodies to take up the concerns that are advanced by a critical perspective. So, of course, we need people to represent and to further develop this critical approach and to defend these ideas, but we favour the idea of trying to "convert" MHP rather than to combat it in a battle that may result in MHP entrenching itself in its "uncritical" positions.

To summarise, our position is that we need critical health psychologists, rather than critical health psychology. To broaden this idea for a moment, we are convinced that a critical perspective is an essential aspect of every sound scientific undertaking, psychological or other (medical, economical...). Furthermore, what a critical approach to health psychology is teaching us today should not to be conceived as "This is CHP”, because tomorrow, the same critical perspective may (and hopefully will) direct our attention to completely different things. Science, also psychological science, is like a person, and, for that matter, like nature: it is always in motion. 
As far as the specific situation of public health psychology is concerned: yes, we have to be especially concerned with the poor, the underprivileged, we have to be culturally sensitive, and use interpretative methods, and we should also work with the systems and institutions (economical, political, cultural...) that control people's environment and in this way influence health and welfare, and we should be prepared to somehow engage in action. All these concerns are rightly underlined by critical health psychologists. However, while we do not consider ourselves explicitly as such, we are not unaware of the importance of these aspects and we, like many colleagues in MHP, have been trying to incorporate them in our professional activities, both in applied public health psychology and applied health promotion. Given this experience, however, we must agree with Julie (and CHP) that, while important, this is also a very difficult task, one that we are not well prepared for. But again, we feel that an explicit oppositional approach by CHP will here as well reduce the possibilities for constructive collaboration with others involved, rather than facilitating them. And if we as health psychologist cannot stimulate each other through cooperation, it will become increasingly difficult to gain the support of the political and economical actors, without whom we will never win the battle to ameliorate the health and welfare of populations.

Hepworth, J. (2006, in press). "The emergence of Critical Health Psychology: Can it contribute to promoting Public Health?" Journal of Health Psychology 11(3).

Smith, T. W. and J. Suls (2004). "Introduction to the special section on the future of health psychology." Health Psychology 23(2): 115-8. 